Particle Accelerators, 1990, Vol. 28, pp. 161-168 Reprints available directly from the publisher Photocopying permitted by license only
(C) 1990 Gordon and Breach, Science Publishers, Inc. Printed in the United States of America

\title{
PERFORMANCE OF THE SUPERCONDUCTING MAGNETS FOR THE HERA ACCELERATOR
}

H. R. Barton Jr., R. Bouchard, Yanfang Bi, H. Brück,

M. Dabrowska, Wenliang He, Zhuomin Chen, Zhengkuan Jiao,

D. Gal1, G. Knies, J. Krzywinski, J. Kulka, Liangzhen Lin,

A. Makulski, R. Meinke, F. Müller, K. Nesteruk, J. Nogiec,

H. Preißner, P. Schmüser, Z. Skotniczny, M. Surala, Wenlong Shi

Deutsches Elektronen-Synchrotron DESY, 2000 Hamburg 52, Germany

Abstract A11 superconducting dipole and quadrupole magnets for the proton-electron collider HERA are subjected to extensive cold tests comprising measurements on maximum field capability, field integral, field direction and field uniformity.

The magnets measured so far exceed the design performance by a safe margin. Special attention is devoted to the field distortions caused by persistent eddy currents and to their time dependence.

\section{INTRODUCTION}

The proton storage ring of the proton-electron collider HERA /1/ presently under construction at DESY in Hamburg is equipped with 422 superconducting dipole and 224 quadrupole magnets $/ 2,3 /$. For the nominal proton energy of $820 \mathrm{GeV}$ the necessary dipole fields of 4.68 $T$ and quadrupole gradients of $91.2 \mathrm{~T} / \mathrm{m}$ are achieved with a current of $5027 \mathrm{~A}$ in the coils. The magnetic length is $8.8 \mathrm{~m}$ for the dipoles and $1.9 \mathrm{~m}$ for the quadrupoles.

At the low injection energy of $40 \mathrm{GeV}$, the magnets suffer from severe field distortions caused by persistent eddy currents in the superconductor which are induced when the main field is changed. In addition to the well-known sextupole field in the dipoles, higher-order multipoles are non-negligible: a 10-pole in the dipole and a 12-pole in the quadrupole. Superconducting correction coils are mounted on the beam pipe of the magnets to compensate the field distortions. The tune of the machine is adjusted by quadrupole correction coils which are wound on top of the sextupole coils. Closed orbit distortions are corrected by dipoles which are installed in the quadrupole cryostats.

The industrial production of the superconducting magnets is well underway. A11 main quadrupoles have been produced and about 200 have been measured. More than 60 dipoles have been delivered and measured. Half of the dipoles are produced in Italy, the other half in Germany. The quadrupole production has been split between France and Germany. 


\section{MEASUREMENTS ON MAGNET PERFORMANCE}

\section{Measurements Methods}

In the dipole magnets, the field integral and direction are measured with a detector /4/ containing a nuclear magnetic resonance (NMR) probe and two orthogonal Hall probes which are rigidly coupled to an electronic tilt sensor.

The field strength in the homogeneous part of the magnet is measured with the NMR probe in steps of $25 \mathrm{~cm}$, the end field is determined with the Hall probes in steps of $2 \mathrm{~cm}$. The overall accuracy on the field integral is better than $1 \cdot 10^{-4}$. The combination of orthogonal Hall probes and tilt sensor allows to measure the field direction with respect to gravity with a precision of 0.2 mrad.

In the quadrupole magnets the same quantities are obtained from a "stretched-wire" system /5/. A single wire, stretched along the quadrupole axis, is precisely moved in the horizontal or vertical direction and the resulting flux change is recorded by means of a sensitive amplifier and a voltage-to-frequency converter /6/. The integrated quadrupole gradient is determined with a accuracy of $5 \cdot 10^{-4}$, the field direction to within $0.3 \mathrm{mrad}$ and the quadrupole axis to within $0.2 \mathrm{~mm}$.

The multipole components in both types of magnets are measured with rotating pick-up coils /7/ which are designed to compensate the large induced signal from the main pole. The accuracy for higher order multipoles is $1 \cdot 10^{-5}$ relative to the main pole. Multipole measurements at room temperature are performed with an alternating current of $11 \mathrm{~Hz}$ and a lock-in amplifier.

\section{Maximum Field Capability}

A11 HERA magnets are quenched a number of times to investigate their training properties and maximum current capability. The magnets show basically no training; more than $80 \%$ reach the critical current of the cable already in the first quench. The quench current distribution at a helium temperature of $4.75 \mathrm{~K}$ is shown in Fig. 1. The values measured in the dipoles are lower than in the quadrupoles because of higher local fields in the coils. Not a single quench has been observed below the nominal current of $5027 \mathrm{~A}$. With one exception, a11 

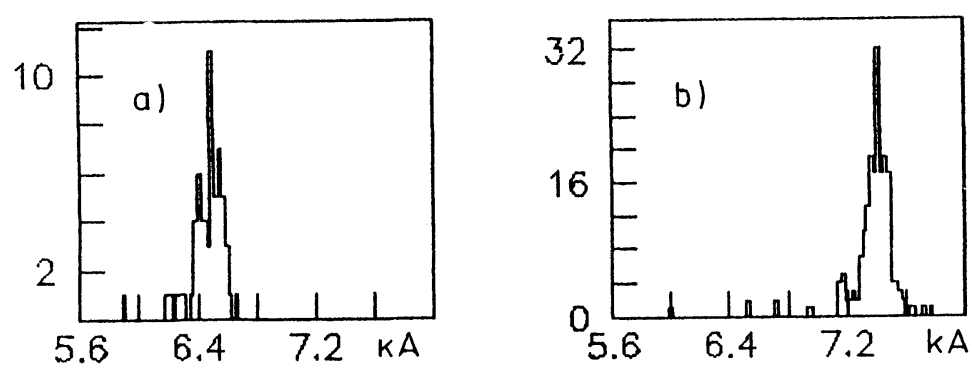

FIGURE 1 Distribution of maximum quench currents for dipoles (a) and quadrupoles (b).

magnets measured so far would allow to operate the HERA proton ring up to an energy of $1 \mathrm{TeV}$.

\section{Field Integral and Field Direction}

The integrated dipole fields and quadrupole gradients, normalized to the magnet current, are shown in Fig. 2. Both distributions are quite
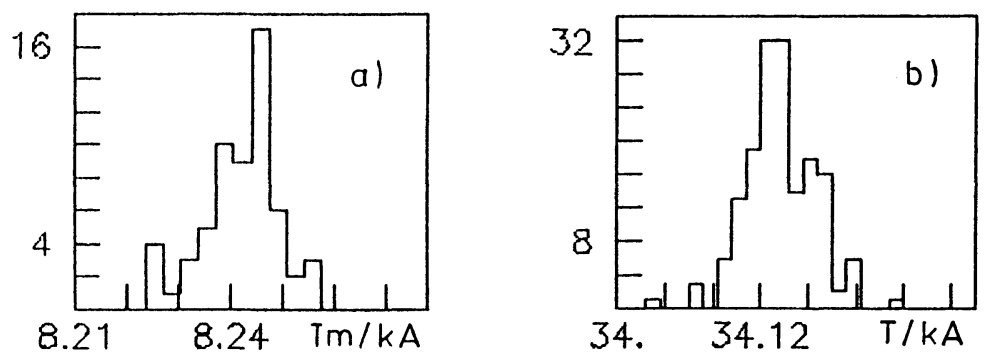

FIGURE 2 Integrated dipole fields (a) and quadrupole gradients (b), devided by the current I ( $I=2100 \mathrm{~A}$ for dipoles and $\mathrm{I}=5000 \mathrm{~A}$ for quadrupoles).

narrow, $0.3 \cdot 10^{-3}$ for the dipoles and $0.6 \cdot 10^{-3}$ for the quadrupoles. In the magnetic lengths, a systematic difference of $1 \cdot 10^{-3}$ is observed between the dipoles of the German and the Italian production and between the quadrupoles of the German and the French production.Fig. 3

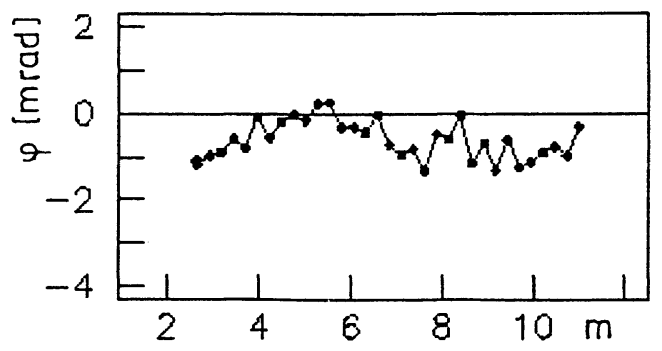

FIGURE 3 Field direction with respect to gravity along the axis of a dipole magnet. 
shows the variation of the field direction of a dipole along the magnet axis. For most magnets, the fluctuations are well within the specified limits of \pm 3 mrad.

\section{Harmonic Analysis of Field Uniformity}

The field of an accelerator magnet is conveniently expressed in terms of a multipole expansion in a cylindrical coordinate system $(r, \theta, z)$

$$
B_{\theta}(r, \theta)=B_{r e f} \sum_{n=1}^{\infty}\left(\frac{r}{r_{0}}\right)^{n-1}\left(b_{n} \cos (n \theta)+a_{n} \sin (n \theta)\right)
$$

and $B_{r}$ correspondingly. Here $r_{0}=25 \mathrm{~mm}$ is the reference radius of the expansion and $B_{\text {ref }}$ the amplitude of the main field component at the reference radius. The $b_{n}$ and $a_{n}$ are the normal and skew multipole coefficients.

The magnet coils without yoke are measured at room temperature during the industrial production process. The complete magnets are measured in the DESY test facility in the superconducting state.

The $b_{n}$ multipoles from "warm" and "cold" measurements of dipoles and quadrupoles are shown in Fig. 4 together with their rms standard
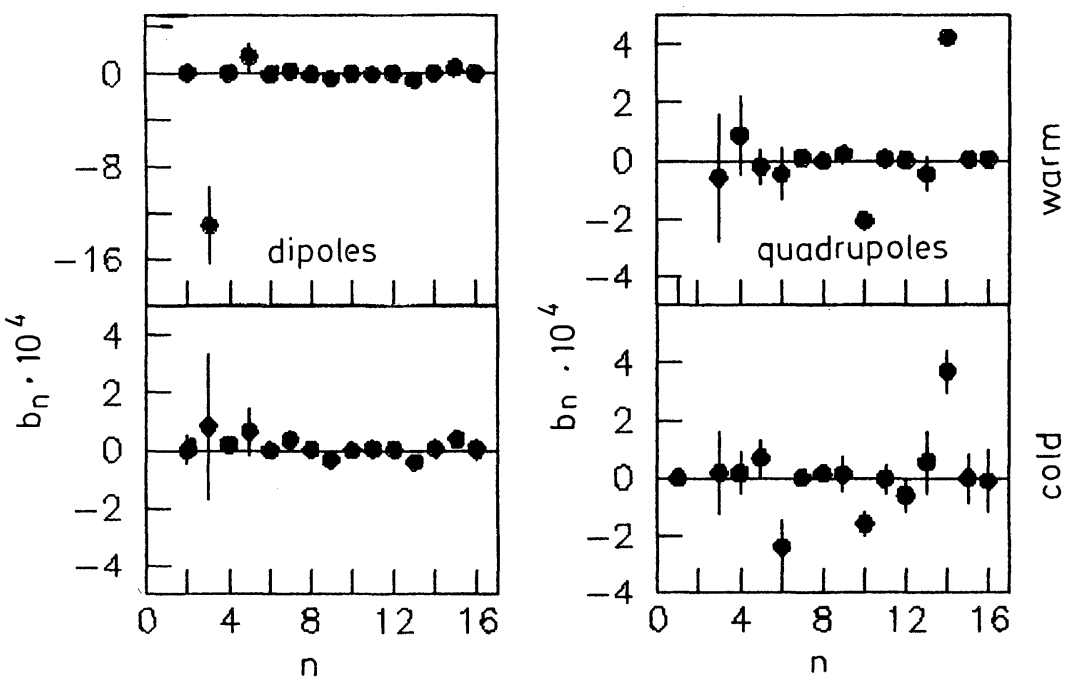

FIGURE 4 Multipole coefficients $b_{n}$ of dipoles and quadrupoles from "warm" measurements, and"at 5000 A ("cold").

deviations. The observed field distortions are very small and well within the allowed limits of $\pm 3 \cdot 10^{-4}$ for the dipoles. Fig. 5 demonstrates that a correlation exists between the sextupoles 


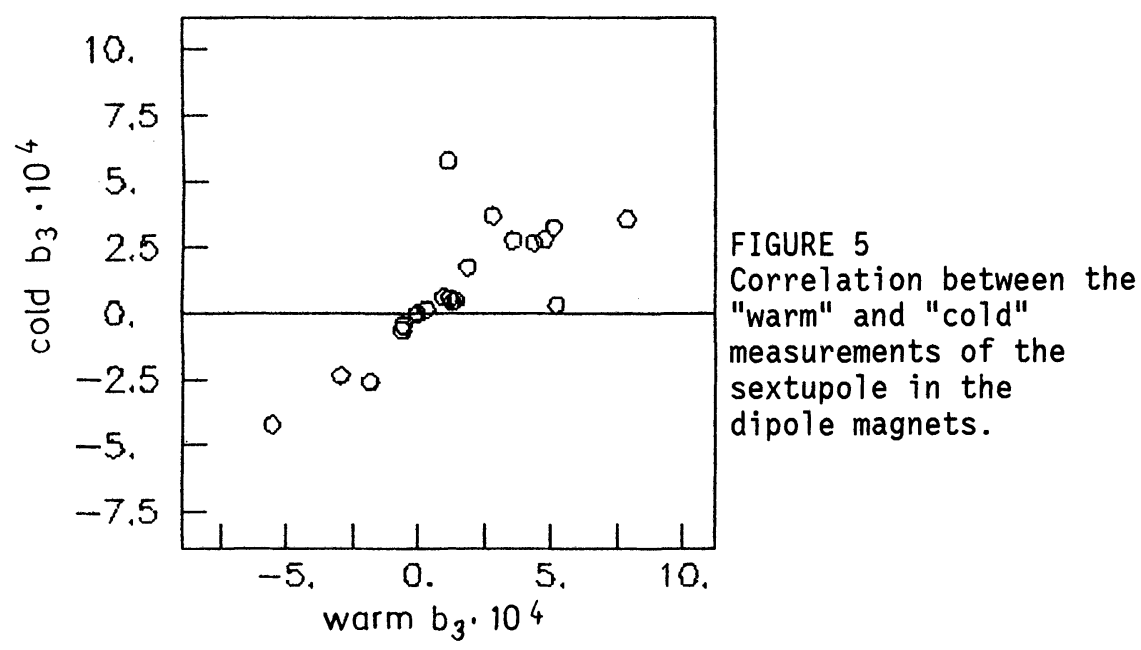

measured for warm coils and those for complete magnets in the superconducting state. A systematic difference of $13 \cdot 10^{-4}$, due to the contribution of the iron yoke, has been subtracted.

\section{Effects from Persistent Currents}

The persistent eddy currents which are induced in the niobiumtitanium filaments during ramping have opposite direction increasing and decreasing main field. The resulting hysteresis is clearly observed in the 6- and 10-pole coeffients of the dipoles and the 12and 20-pole coefficients of the quadrupoles (Fig. 6). The predictions of an eddy current model /8/ are shown as continuous curves in Fig. 6. The agreement with the data is remarkable.

The persistent currents have also a significant influence on the main field component of the magnets. Their contribution is deduced by taking the difference between the measured dipole or quadrupole fields and the field values computed from measurements at higher currents. The data are shown in Fig. 7, normalized to the computed field values. A similar hysteresis as in the multipole data is observed and again good agreement with the model prediction.

\section{Time Dependence of Persistent Current Effects}

All multipole fields which are generated by the persistent eddy currents decrease with time. For the accelerator this effect is most severe during the injection of the proton beam which takes about 30 minutes. Time dependence measurements are performed at $250 \mathrm{~A}$, 


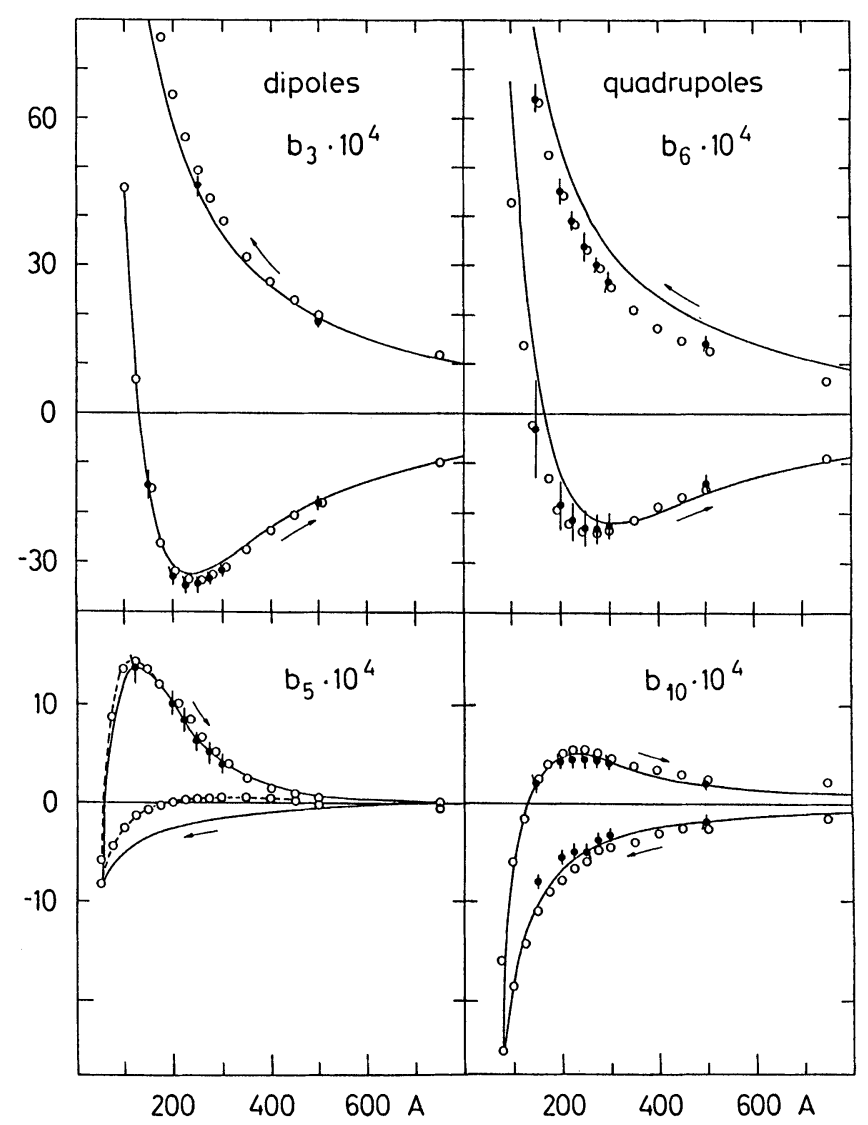

FIGURE 6 Hysteresis measurements of higher multipoles in dipoles and in quadrupoles. Open points: 1 magnet. Full points: average of 19 dipoles ( 33 quadrupoles). Here, the transport current contribution has been subtracted. The curves are model calculations (see text).

following an initial current cycle $50 \mathrm{~A} \rightarrow 6000 \mathrm{~A} \rightarrow 50 \mathrm{~A} \rightarrow 250 \mathrm{~A}$. The absolute values of the sextupole in a dipole and of the main pole are plotted in Fig. 8 against the logarithm of the time elapsed since the current of $250 \mathrm{~A}$ had been reached. In both cases an almost linear drop is observed between $100 \mathrm{~s}$ and $3000 \mathrm{~s}$ and some flattening outside this region. The deviation from linearity at low $t$ is subject to the choice of the time origin. In all measurements we find that the low $t$ points can be fitted too if a form $\log \left(t+t_{0}\right)$ is chosen with $t_{0}$ as a free parameter. More details will given elsewhere /9/. The resolution of the stretched-wire system was not sufficient to establish a time variation in the quadrupole gradient.

our data indicate that the time variation is different for 

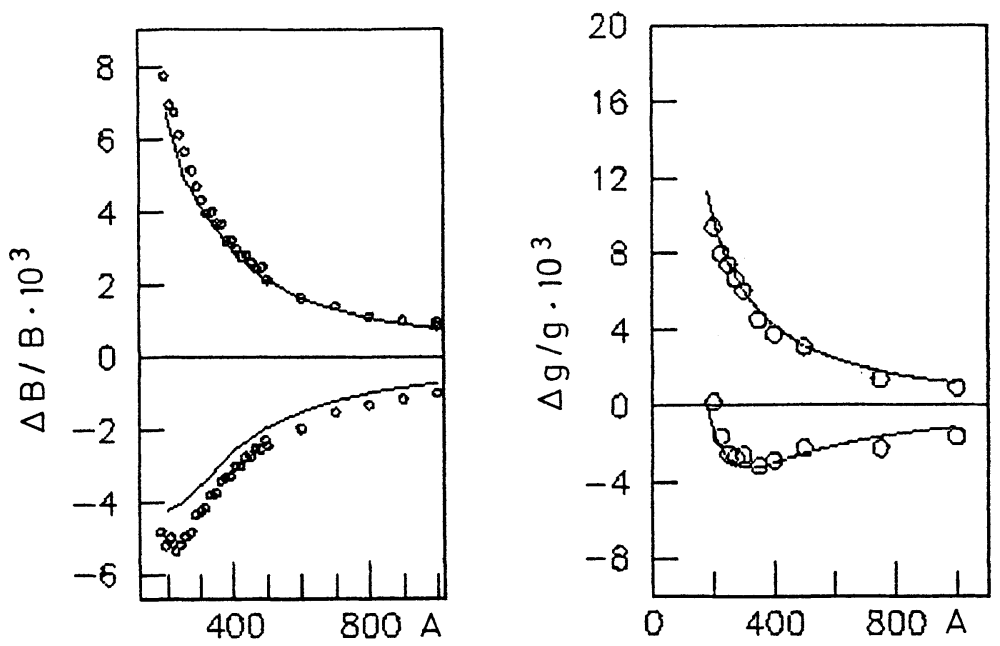

FIGURE 7 Hysteresis measurement of main fields: $\triangle B / B$ in a dipole (a) and $\Delta g / g$ in a quadrupole (b), as explained in the text. Curves: model prediction
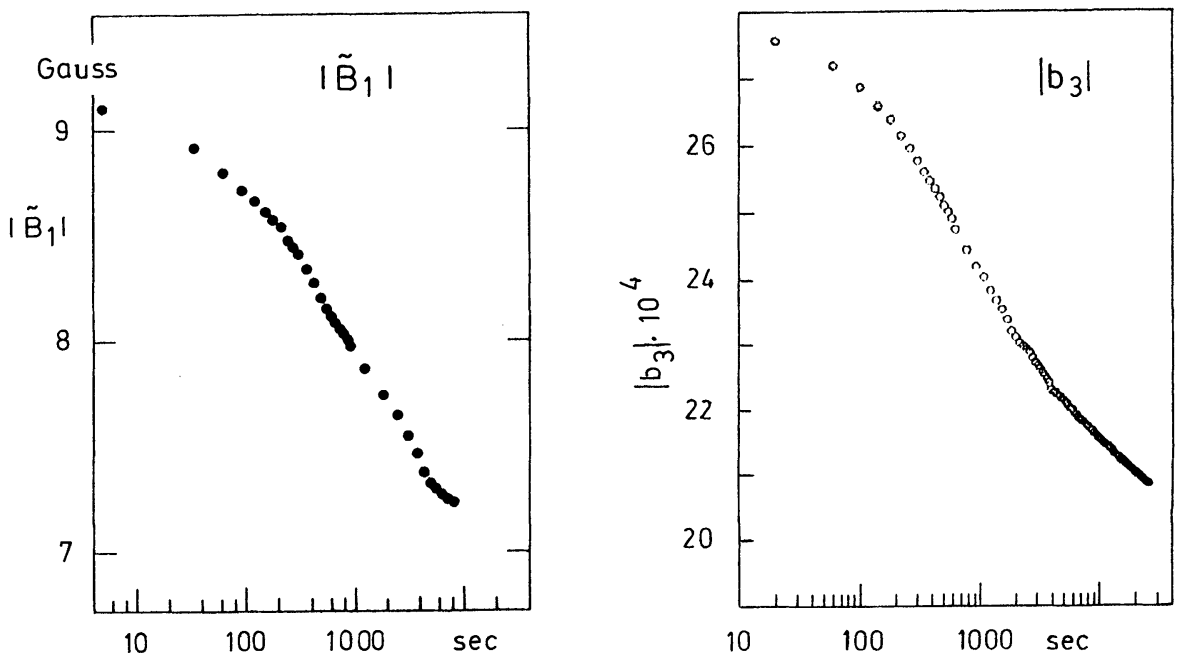

FIGURE 8 Time dependence of the absolute value of the eddy current contributions to the main field and to the sextupole in a dipole, on $a \log (t)$ scale with $t$ measured since end of ramping.

different superconducting cables. In the German dipoles, which are made from $A B B$ conductor, the persistent current contribution to the dipole and sextupole fields changes by about $6 \%$ within the first 30 minutes. In the Italian dipoles made from LMI conductor, the change 
appears to be twice as high. The lowest variation of about $5 \%$ is found in the 12-pole field of the quadrupoles which are all made from Vacuumschmelze superconductor.

The most likely explanation of the time dependence is flux creep in the superconductor leading to a logarithmic decrease of the critical current density. According to theory /10/ the creep rate is expected to depend critically on the nature of the pinning centers and may therefore be different for conductors from different manufacturing processes.

\section{SUMMARY}

A large number of the superconducting HERA magnets have been tested. The magnets show excellent field quality and quench training behaviour allowing operation of HERA with a large safety margin. Persistent eddy currents lead to time dependent field distortions at low energies which have to be compensated by correction coils.

\section{ACKNOWLEDGEMENTS}

We thank all members of the measuring group for their efforts in data taking and the staff of the DESY refrigerator plant for the regular supply with liquid helium to operate the magnet test facility.

\section{REFERENCES}

/1/ For a recent review see:

B. Wiik, Proceedings of the XXIV Intern. Conf. on High Energy Physics, Munich 1988

12/ For recent reviews see:

H. Kaiser, 13th Intern. Conf. on High Energy Accelerators; Novosibirsk, USSR, Aug. 1986

S. Wolff, MT-10, Boston, Massachusetts, USA, Sept. 1987

/3/ R. Auzolle et a1., ICFA Workshop on Superconducting Magnets and Cryogenics, BNL 52006, p. 195, May 1986

/4/ A. Makulski et al. to be published

$/ 5 / \mathrm{G}$. Knies et al. to be published

/6/ G. Hase et a1., DESY report HERA 87-23 (1987)

/7/ H. Brück et al. to be published

/8/ H. Brück et al., Field Distortions from Persistent Currents in the Superconducting HERA magnets, DESY Report 89-41 and

Z. Phys. C, to be published

19/ H. Brück et al., Time Dependence of Eddy Current Effects in the Superconducting HERA Magnets, MT-11, Tsukuba, Japan 1989

/10/ P.W. Anderson, Phys. Rev. Letters 9,309 (1962)

P.W. Anderson and Y.B. Kim, Rev. Mod. Phys. 36, 39 (1964)

M.R. Beasley et a1., Phys. Rev. 81, 682 (1969) 